Research Article

\title{
Optimization of Hybrid Multimedia Art and Design Teaching Mode in the Era of Big Data
}

\author{
Hua Tian \\ Shanxi Technology and Business College, Taiyuan 030006, China \\ Correspondence should be addressed to Hua Tian; tianhua122184720@163.com
}

Received 4 September 2021; Revised 27 September 2021; Accepted 9 November 2021; Published 17 December 2021

Academic Editor: Tongguang $\mathrm{Ni}$

Copyright (C) 2021 Hua Tian. This is an open access article distributed under the Creative Commons Attribution License, which permits unrestricted use, distribution, and reproduction in any medium, provided the original work is properly cited.

\begin{abstract}
In this paper, a 9-layer convolutional neural network with 4 convolutional layers, 4 pooling layers, and 1 fully connected layer is designed to recognize the emotions of digital learning images in the era of big data. The convolutional neural network is trained using digital learning images that have been labeled with emotions, and the final test shows that the network has good recognition results. This in turn causes the information overload problem to arise. And combined with the questionnaire results and interviews, it was found that there are problems of technology for technology's sake, teaching for teaching's sake, and in multimedia teaching, and these will add to the psychological and visual sensory burden of students and easily cause the information overload problem. The types of information overload problems in multimedia-assisted teaching are summarized as follows: unreasonable presentation of information, which causes audiovisual redundancy; too much teaching irrelevant information, which increases the external cognitive load; and an uncoordinated audiovisual environment, which increases the external cognitive load. Starting from the perspective of the integration of preservice to in-service art teachers' new media art curriculum design and teaching ability development, three representative teacher education cases were studied using a combination of teaching practice and case tracking methods to summarize the successful experiences and effective ways of art teachers' new media art curriculum development and teaching ability development, which will provide future art teacher training and in-service teachers' professional development. Both are below 5\%. The types of funny emotions are mainly distributed in animation teaching methods. Animation resources are generally well designed in color and layout and can convey good visual emotional characteristics. In other types of images, the emotional distribution level of funny is less than $10 \%$. It is worthwhile to learn from this experience.
\end{abstract}

\section{Introduction}

After entering the era of big data, human beings will face more impacts between big data and culture, artificial intelligence is developing rapidly, the world culture, technology, and art are facing new challenges, and images are flooding our lives like never before. However, the private demolition of cultural and historical buildings to build mansions, the piracy of famous Parisian architectural communities, the construction of rehashed Egyptian pyramids, and the placement of indecent public sculptures, all these ugly cultural images reflect the lack of people's aesthetic level, cultural respect, and creative consciousness, repeatedly becoming an obstacle to the development of civilization [1]. The development of civilization cannot be reduced to the sacrifice of science and technology. The aesthetics and inner cultural beliefs of human beings are the important force of the development of the times. In contrast, the current situation of art museum education in China is that art museum education activities are in various forms, but they lack the depth and extension of education and do not play a role in improving the visual literacy of citizens [2]. This study aims to explore the current situation of art museum education, understand the impact of art museum education on students' visual literacy, explore how art museum education should promote the improvement of students' visual literacy in the context of the big data era, cultivate a generation of citizens with good artistic cultivation, and promote the development of a generation of civilization progress [3]. The advent of the big data era has 
exposed a large amount of information to life, impacting modern human attitudes of thought and visual perception. Two hot zone comparison pictures with very strong synthesis, very weak sequence type, very weak synthesis type, and very strong sequence type are referred to as comprehensive type and sequence type hereinafter. The large volume and varying quality of information affect human cognition while testing modern humans' discernment and aesthetic ability of current information. The rapid development of social civilization is often hindered by the level of human's aesthetic cognition, such as the out-of-place urban sculptures and the high ratings of vulgar films and TV shows, which reflect the lack of quality of life and the pursuit of "beauty" in the current impetuous and fast life of human beings. This is the downside of the rapid development of material civilization and the difficulty of the public's visual literacy and civilizational awareness to keep up with the development of civilization.

The rapid development of information technology has not only revolutionized our work, study, and life but has also had a profound impact on the field of education and teaching, greatly contributing to the improvement of the efficiency of teaching and learning in schools [4]. In recent years, the way of teaching and learning in schools has also undergone major changes with the advancement of technology. With the continuous reform and innovation of technology, we have achieved a change from the early days of using audiovisual and auditory media such as slide projectors, video visualizers, tape recorders, radios, and other teaching devices to the present-day application of interactive whiteboards, multimedia computers, and network teaching platforms. The application of these devices and related teaching software in teaching has not only changed the effect of face-to-face teaching but also made it possible to realize distance network teaching across the limitation of space and distance. Based on the characteristics of the Internet itself, online network teaching is more informative, richer in learning resources, and more interactive in real time [5]. The comprehensive learning style is more interested in graphic information. When studying, he can integrate graphic information more closely, and the reading time is shorter. Participants with strong sequence type basically followed their logic when reading text. And with the continuous advancement and innovation of artificial intelligence, big data, cloud computing, and other information technologies, the impact of technology on the traditional classroom and changes to the field of education will grow, thus benefiting more schools and teachers and students [6]. Special emphasis is placed on the important role of the modern media arts curriculum in developing students' core literacy in the subject. Accordingly, how to give full play to the teacher's role in achieving effective teaching and better development of subject core literacy within the limited class time also becomes an important issue that must be properly addressed in teaching. In this study, the author tries to put the unit teaching "zero into whole" design concept throughout the modern media art course and strives for more focused teaching objectives, more optimized teaching content, and more comprehensive student abilities [7].
In today's booming modern information technology, the human scientific process is developing at an unprecedented speed, and the power of increasingly advanced information technology for film and television art is also more profound. As the seventh category of art, film and television art is different from painting, music, theater, and other ancient art forms, it has been accompanied by the process of science and technology since its birth, and film and television have a high degree of integration with modern information technology and tacit understanding. Modern information technology has pushed film and television art to take a big step towards industrialization, and it is also under the high degree of integration and interaction between information technology and film and television art that digitalization, virtualization, high speed, technology, specialization, and internationalization of film and television art have been realized, and information technology has had a profound impact on the creation, dissemination, and consumption of film and television, as well as on the aesthetics of film and television. This is the most favorable era for the development of the film and television industry, and modern information technology is also in the era of becoming the strongest development, which is the best era in human history, but at the same time the worst era. Under the influence of modern information technology, does film and television art grasp the most essential form of realization of film and television art? Without the help of modern information technology, will film and television art lower its level, or even lose its audience and box office? Film and television art as the seventh category of art forms has been in the form of cutting-edge and rapid performance. As the audience, we are undoubtedly experiencing great changes, and this change is also happening under the influence of modern information technology. The closer the combination of the two, the greater the mutual force will be. The content of both sides is constantly changing, the form is changing as well, and the audience's experience is also changing. The film and television art both technically and artistically has produced great fluctuation and impact.

\section{Current Status of Research}

Research on blended learning is much earlier abroad than at home. At present, the research on blended learning is mainly reflected in the concept and how it is applied. It is a local theory born and grown in China, and the research is still in the initial stage [8]. After the theory was proposed, a seminar on multimedia picture language was held at Tianjin Normal University. Since picture art design before that mostly applied to other disciplines of art theories, Lande et al. proposed a multimedia picture language art theory, which solved the problem of borrowing other disciplines of art theories [9]. After that, Li combined multimedia picture art theory with cognitive psychology to study the cognitive law of multimedia picture language and explored the cognitive law that should be followed in the design of multimedia teaching resources [10]. Patel and Sharma further developed 
multimedia picture language in "Research on the Construction of the Theoretical System of Multimedia Picture Linguistics," drawing on other linguistics, psychology, educational technology, and other related achievements, which provides a reference for multimedia picture language subsequent research providing a referenceable research framework as well as ideas and methods [11]. The strategy emphasizes three major educational resources, namely, educational policy and planning, teacher development and information and communication technology, and four priority development areas, namely, literacy, vocational and technical education and training, higher education, and learning process and outcome enhancement, and three major learning activities, namely, global citizenship education, education for sustainable development, and health education, intending to promote quality and comprehensive lifelong learning and thus fostering creativity and responsibility [12]. How to use multimedia to facilitate human learning is a central question in the study of multimedia learning. Ramsgaard Thomsen et al. divides the study and development of multimedia teaching and learning into two categories: the first category is technology-centered, and the second category is learner-centered [13].

Some foreign language colleges and universities began to have more advanced e-teaching equipment, such as simple language laboratories, and with the rapid development of e-education, e-education equipment was gradually applied to multimedia-assisted teaching. After entering the nineties, China's information technology developed rapidly, and since then, multimedia-assisted teaching has also been developed significantly. The research on multimedia teaching in this period is also more in-depth than that in the previous period, and there are researches on how multimedia teaching is used in specific subjects in colleges and universities and further analyses on how multimedia teaching is used in teaching practice [14]. 51.59\% of the students said that the background color matching of the text in the multimedia courseware is relatively reasonable, which will not cause visual disturbance or redundancy, but $48.41 \%$ of the students said that the multimedia courseware has unreasonable places in the color matching; this will also disturb the learning and cause visual redundancy for students. Along with the increasing progress of education informatization, the use of multimedia-assisted teaching as a modern teaching tool has become increasingly common in the teaching of colleges and universities and has gradually become an important teaching tool in colleges and universities. The educational content in modern media art is provided, but it has certain problems, such as the prevalence of low attention, fragmented teaching content, single teaching method, fewer research results, and lack of relevant knowledge of teachers [15]. In response to these problems, this study uses literature method, questionnaire survey method, and comparative method to study the current situation and problems of the modern media art curriculum, and after analyzing the reasons, based on postmodern curriculum theory, constructivist teaching theory, theory of unified curriculum and interdisciplinary teaching, evaluation theory, multiple intelligence theory, and visual culture theory to compile and program design for new media art in secondary schools [16]. The birth and development of the art of cinema and the evolution of the art of cinema were accomplished in an integrated way with the role of technology: for the emergence and invention of the film camera and projector before the emergence of film as real art, due to technological improvements in the art of film and television added the element of sound, and the development of film technology contributed to the journey of color film, with the technology of film stunts, the Electricity Bureau could produce fantasy. The spectacle nature of cinema only then discovered, and cinema was further released on a deeper level.

In the context of a rapidly developing information society, the development of modern media art has grown rapidly and has fully penetrated all aspects of life, providing a favorable environment and excellent technology for the development of modern media art curriculum and teaching in schools. For the current generation of high school students, their social background and living environment have also resulted in greater interest and enthusiasm for digital products and video art than traditional art media, which is also an intrinsic motivation for the continuous development of modern media art learning modules.

\section{Optimization Analysis of Hybrid Multimedia Art and Design Teaching Mode under Big Data}

3.1. Multimedia Art Design Big Data Design. As one of the main manifestations of multimedia learning resources, the design of digital learning screens and their related research should be based on a theory that is consistent with human learning styles and should be explored and practiced in conjunction with the cognitive theory of multimedia learning. Richard E. Mayer of the University of California proposed a cognitive theory of multimedia learning based on three views of cognitive science and pointed out that multimedia-based learning activities should include three assumptions, namely, the dual-channel assumption, the limited capacity assumption, and the active learning assumption, to explain the cognitive tendency of learners when using multimedia resources for learning. The dualchannel hypothesis refers to the fact that people have separate information processing channels for materials with visual and auditory representations [17]. Paying attention to result evaluation, process evaluation is relatively lacking, and so on. The author also started thinking and researching and believes that modern media art curriculum learning evaluation tools should be developed, and learning evaluation dimensions should be established. In the evaluation, modern media art creation and appreciation capabilities should be integrated, and students should be clear that modern media art is not just creation of works. When visual information such as on-screen text and images containing knowledge, content is delivered to the learner's eyes, and the acquired information is processed in the visual-image channel; when narration, background music, and other information are 
presented to the learner's ears, the acquired information is processed in the auditory-verbal channel. Meyer argues that although the acquired information enters the information system through one channel, the learner can change the processing channel through a switch in representation. For example, when a learner looks at a text, he or she first represents the information through the visual-image channel, but a learner with the ability to read text can convert the information received through the visual into audio information, and the relationship between the two can be transformed through different representations.

The active processing hypothesis refers to the idea that learners actively engage in cognitive processing to build a coherent mental representation of their experience, meaning that learners' activities in processing multimedia information are active rather than passive. Meyer states that the three processes of active processing involve selection, organization, and integration. Selection means noticing multimedia information and creating discourse and picture material; organization means establishing intrinsic connections between the selected knowledge information; and integration means establishing extrinsic connections between the organized verbal model and image model and prior knowledge, thus facilitating meaningful learning to occur. Multimedia images should be composed in a way that is consistent with the subject matter to be represented and with certain artistry, like the art of speaking and presentation. Having a certain art is, for example, aesthetic, but more important is the effectiveness of its expression, which can be explored at the content level, the cognitive level, and the picture structure level in terms of its logical laws. Xiaofeng described the 12 multimedia design principles, which include the multimedia cognition principle, spatial proximity principle, time proximity principle, always principle, channel principle, redundancy principle, and personalization principle [18]. Thereby, this strengthened the harmonious interaction between learner's emotion and picture emotion in the smart learning environment. The coding of the digital learning screen can help users understand the attributes of the screen from the side. However, it is macrolevel, difficult to play a practical role, not yet able to solve the actual problems faced. Multimedia screen language has initially formed its unique design methods and rules based on the rules of interpretive information design, explored the logical main line of screen representation content, and made multimedia design followable from the relationship between media, between media and content, and between media and surrounding environment. The basic framework of big data multimedia screen linguistics theory is shown in Figure 1.

Things are universally connected and are organically linked to each other, interdependent and mutually constraining; there are no things that exist in isolation from the surrounding things and conditions. Therefore, in home education statistics, in a particular study not only the thing itself, but also other things that interconnected with it, and find the connection that exists between them through reasonable ways and means. The interdependence between things or phenomena can be broadly divided into two kinds: one is the function relationship, which is a kind of deterministic relationship; that is, when the value of a thing changes, the value of its related things or phenomena will also change accordingly. There is also a correlation, which means that there is a certain relationship between things, which cannot be described by a fixed causal relationship, and at the same time, although the correlation is not definite, it is regular.

Both regression analysis and correlation analysis can be used to describe correlations, and both can measure the relationship between two or more variables to determine the relationship between them. This is what regression analysis and correlation analysis have in common. However, broadly speaking, regression analysis is subordinate to correlation analysis, and strictly speaking, there is a difference between the two. The purpose of correlation analysis is to measure the closeness of the relationship between two random variables. Regression analysis, on the other hand, is more oriented towards changes between variables, describing their relationship through mathematical expressions and thus determining the extent to which a change in one or several variables affects another identified variable [19]. Using multidistance regression analysis, it is possible to determine how closely one independent variable and the dependent variable are related and to detect factors that are less well related, but whether to discard the worst independent variable or whether that independent variable is at all users cannot be simply based on the results of the mathematical treatment and should be considered holistically based on expertise. The current study established the effect of the blended learning experience as the dependent variable and the corresponding independent variables constructed from the blended classroom process experience and closely related to the multimedia screen, and the equation of the multiple regression was expressed as follows:

$$
\begin{aligned}
Y & =b_{0}-b_{1} x_{1}-b_{2} x_{2}-\cdots-b_{i} x_{i}, \\
Y_{i} & =X_{i} \beta-u_{i} .
\end{aligned}
$$

Multiple linear regression can use ordinary least squares, maximum likelihood, or distance to estimate the parameters, provided the underlying assumptions met. After the regression function is obtained with the data, statistical tests need to be performed on the sample regression function to determine the reliability of the estimates, and the statistical tests that need to be performed include goodness-of-fit tests, tests of overall linearity of the equations for significance, and tests of significance of the variables:

$$
R=\sqrt{\frac{\mathrm{ESS}}{\mathrm{TSS}}} .
$$

The prediction of user ratings can be viewed as an act of filling in missing values for a residual user-item rating matrix; that is, given a sparse matrix where the rows are items, the columns are users, and the specific values of the matrix are user-item ratings, the matrix is constructed to fill in the remaining vacant parts to obtain the user-item ratings, assuming a low-rank matrix, the user-item rating prediction is transformed into a minimization problem. 


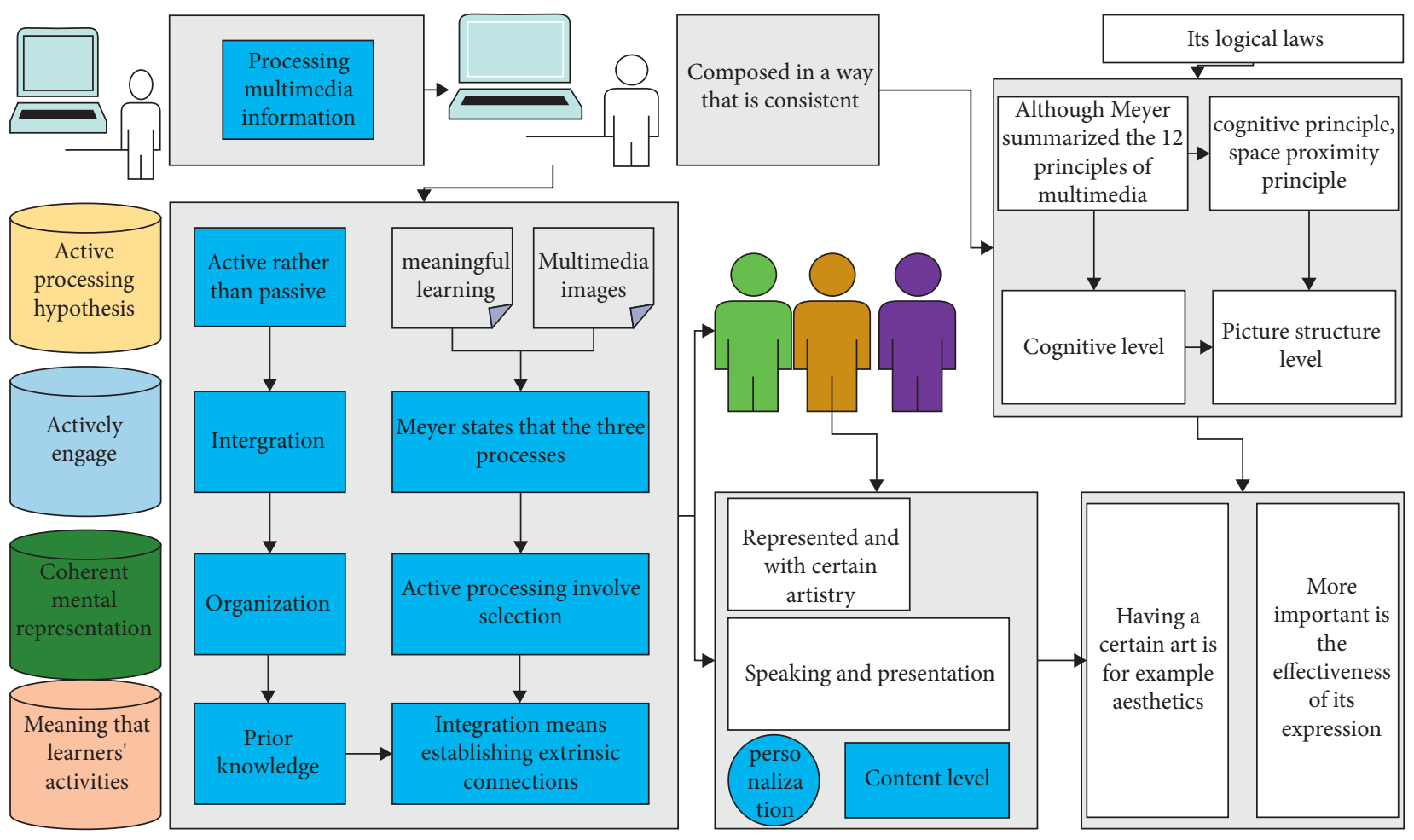

Figure 1: Multimedia art and design big data framework.

$$
\begin{aligned}
& \arg \max \left\|X_{i}\right\|^{e}-\left\|X_{i}\right\|^{2}+\frac{a}{2}\|w(X+Y)\|^{e}, \\
& \arg \max \left\|X_{i}\right\|^{2}+\left\|X_{i}\right\|^{2}-\frac{a}{2}\|w(X+Y)\|^{e} .
\end{aligned}
$$

Large classes are the main form of teaching in China at present, with an average of fifty students per class in primary and secondary schools, and as many as fifty to sixty in colleges and universities, and as few as twenty to thirty, so it is difficult to realize that only one student plays the role of lecturer in each class, but it is possible to work together in small groups to teach a topic. At the same time, conditions should be created for the subordinate information management system to be extended and refined based on this classification system. When students choose a topic, due to the limitations of their knowledge and ability, they need to ask the teacher for advice or learn systematically through the Internet, and so on. Therefore, the hybrid teaching mode needs to be combined with other teaching modes, and collaborative learning and peer-administered teaching can effectively support the hybrid teaching model.

$$
\delta=\sum_{i \in U, j \in U}^{R} \sqrt{p_{i}(r) p_{j}(r) .}
$$

To ensure the accuracy and comprehensiveness of the prediction scores, the prediction results of the blending steps are mixed to obtain the final prediction score matrix.

$$
\varphi_{i}=\frac{1}{1-e^{-\pi i}}
$$

Teaching and learning can be counterproductive. Therefore, teachers need to do a lot of preparatory work when implementing blended learning models. First is that the peer-administered content should be selected considering both the ability level of the giver and the recipient. Second, they should be good at identifying students' strengths and weaknesses. Third is to determine the means of instruction based on the class specifics and the instructional goals of the course. Fourth, the teacher should monitor and guide the entire process, and after assigning tasks to the students, he or she should frequently learn about their progress and guide and supervise them. Fifth is to conduct reasonable teaching evaluation, not only to complete the dissemination of knowledge, but also to focus on the process of comprehensive ability improvement so that this evaluation should serve to check the effectiveness of teaching, but also protect the students' motivation, as shown in Figure 2.

The personalized recommendation method based on potential factors incorporates methods such as matrix decomposition complementation, which not only considers the potential factors of users and items but also considers the respective neighbor information sets of users and items when performing matrix complementation. First, the user rating matrix is constructed, and the user potential factor information and the project potential factor information are modeled and stored in the HBase database, and the user neighbor information set and the project neighbor information set are derived using the similarity calculation formula and stored in HBase; then the original user project rating matrix is matrix-decomposed to obtain the preliminary prediction rating matrix 


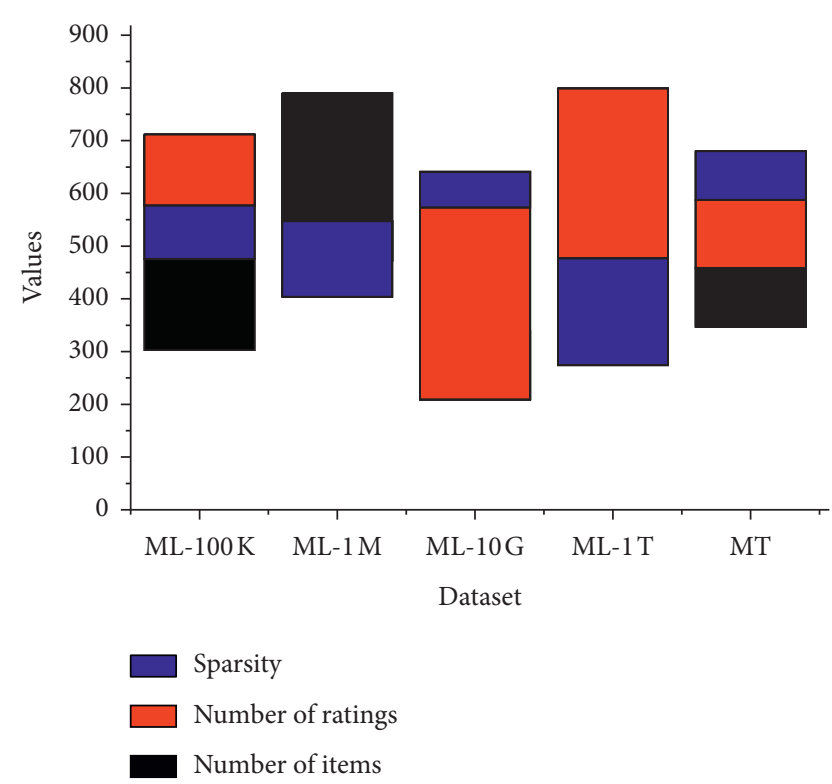

Figure 2: Big data dataset characteristics.

and the true prediction rating error value and stored in the database table. The scoring error values are stored in HBase as database tables.

3.2. Hybrid Multimedia Art and Design Teaching Mode Optimization Design. The principle of systematicity requires that when classifying digital learning images, it is necessary to determine their different subordination and order according to the relevant links of their essential properties so that all digital learning images form a reasonable, orderly, and hierarchical classification system among themselves. The classification of digital learning screens guided by the principle of systematicity helps teachers and researchers to clarify the hierarchical relationship between different screens and their essential properties, which helps to improve the efficiency of classification. The improvement of visual literacy can promote the faster and better development of civilization. Extensibility means setting up shelter categories to ensure that when new things or concepts are added, the established classification system is not disrupted, and it should also create conditions for the lower-level information management system to extend and refine based on this classification system. In other words, the classification of digital learning screens is changed after the design is completed, and small-scale adjustments and changes should be made to the classification rules promptly when there are screens whose essential properties are completely different from the previous classification system or have subordinate relationships.

Practicality requires that the classification criteria for digital learning screens should be such that the actual needs of all relevant units in the system are met as much as possible while meeting the total tasks and requirements of the system. In this study, the classification of digital learning screens developed should first meet the organization and arrangement of all screens based on local disks. The second is to support the subsequent work of this study to achieve accurate identification of digital learning screen emotions, thus enhancing the harmonious interaction between learner emotions and screen emotions in the intelligent learning environment. The encoding of a digital learning picture can help the user to understand the attributes that the picture possesses from the side. For example, a simple encoding can identify the characteristics of the subject to which the screen belongs, the school section to which it belongs, and so on, and facilitate quick retrieval and classification in the computer. In addition, as the database of digital learning screens becomes larger and larger, the need for coding comes to the fore; for example, a standard and stable coding strategy helps to improve the accuracy of classification, the speed of retrieval, and the depth of the database, as shown in Figure 3.

With the rapid development of the Internet, the total amount of digital learning resources has also shown exponential growth. In the research process, it is difficult to manage such many learning images efficiently without a stable and reasonable coding method. With the support of coding, digital learning images with the same properties can be quickly categorized using regular expressions. In addition, when using deep learning to train classification for emotional features of images, the input data can be automatically labeled during the data preprocessing stage to improve training efficiency. Promoting the development of a generation of civilization and progress, the advent of the era of big data has exposed a large amount of information in life, impacting modern people's thinking, attitude, and visual perception.

Sentiment estimation of digitally learned images relies on many digitally learned images as training data, and the amount of training data and labeling accuracy can have an impact on the level of sentiment prediction. In this study, a supervised learning convolutional neural network is used, so image preprocessing such as sentiment labeling of the training data is required before training. According to the previously mentioned emotion-type classification criteria, this study needs to annotate 17,433 digitally learned images according to 14 emotion types: warm, cheerful, lively, funny, exaggerated, humorous, funny, bleak, boring, dull, tedious, unreal, thrilling, and scary, and the annotation intensity is divided into 4 levels, where 0 indicates the lowest intensity and 3 indicates the highest intensity [20]. In this study, 17,433 digital learning images were annotated utilizing paid recruitment of annotators. Except for the necessary training required before the annotation process began, the emotional value of each digital learning image and its intensity calibration depended on the subjective experience of the annotator and was not influenced by the subjective consciousness of the researcher. However, the researcher conducted strict supervision throughout the annotation process to ensure that the entire work was carried out smoothly.

In the modern media course learning, the evaluation link is relatively lacking, and there are also some problems; for example, the evaluation criteria are vague, and the evaluation content is not detailed and clear; the focus on the results of the evaluation, the process of evaluation is lacking. The 


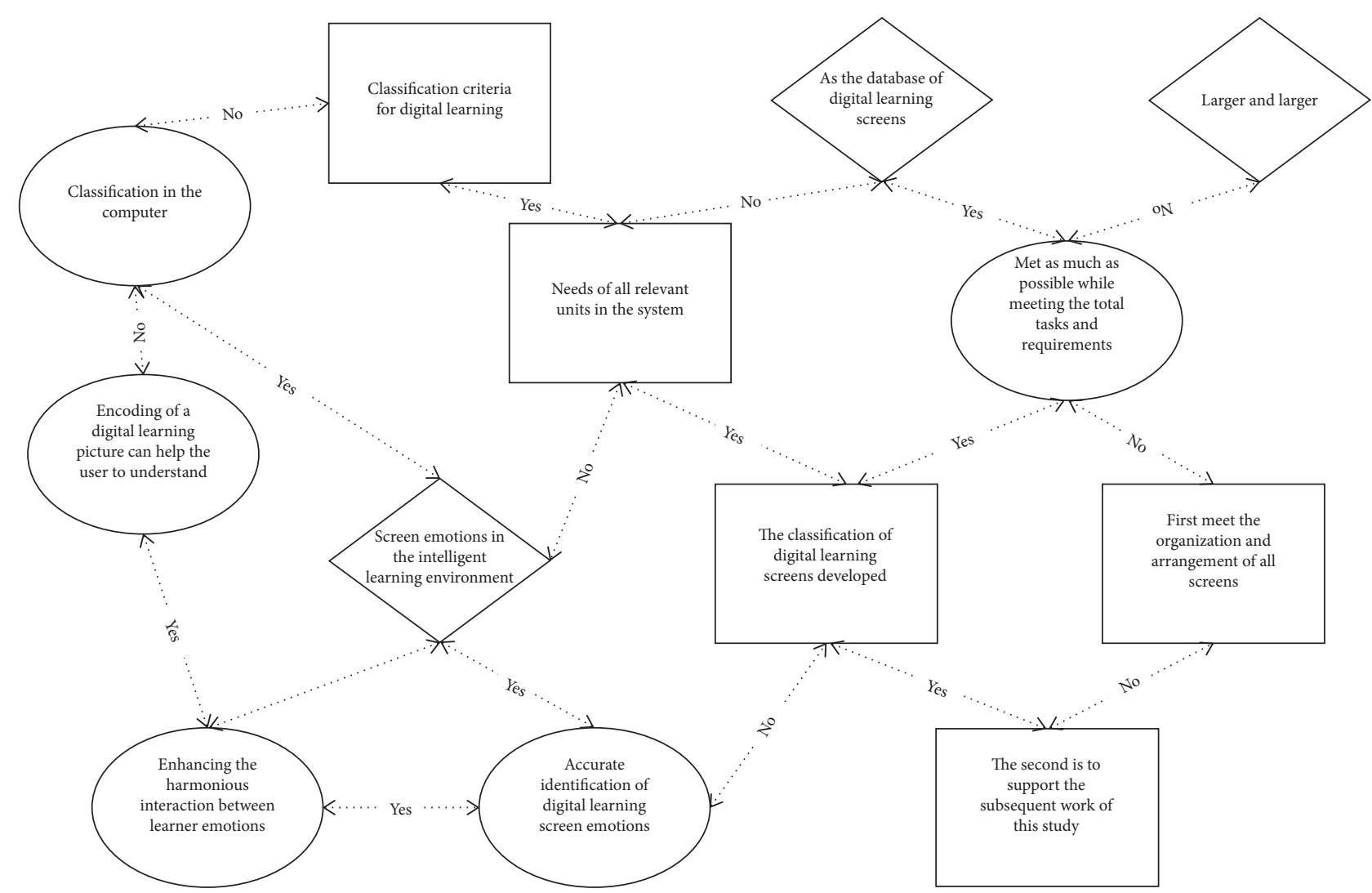

FIgURE 3: Steps to optimize the hybrid multimedia art and design teaching model.

author also started to think and research that modern media art course learning evaluation tools should be developed, set up learning evaluation dimensions, in the evaluation of modern media art creation and appreciation ability into one, guide students to clarify the fact that modern media art is not only the creation of works media, technical changes, but also learning, creation ideology is subversion and innovation of the concept, and advocate a tiered evaluation. The learning assessment rules for the Truth Modern Media Art learning ability level are shown in Figure 4.

Interesting content in multimedia courseware is designed with the initial intention of attracting students' attention in the hope that they can focus on classroom learning. After investigating the interesting but irrelevant content in multimedia courseware, it was found that $31.21 \%$ of the students in this study thought that there was no such irrelevant content that would disturb students' learning [21]. Based on the characteristics of the Internet itself, online network teaching has more information, richer learning resources, and stronger real-time interaction. However, $63.38 \%$ of the students believe that there is content in multimedia courseware that disturbs students' learning. Thus, it seems that the content in multimedia courseware needs to be filtered and not interesting for the sake of being interesting.

$51.59 \%$ of the students said that the matching of text and background colors in multimedia courseware is reasonable and does not cause visual disturbance and redundancy, but $48.41 \%$ of the students said that the color matching of multimedia courseware is unreasonable, which also disturbs learning and causes visual redundancy for students. This shows that beautiful multimedia courseware can attract students' attention, but too much pursuit of gorgeous decoration and neglect of the integration with the content of the courseware will also cause visual disturbance and redundancy; or the courseware is too "plain," the content of the courseware is all words, lack of artistry and technicality of the courseware, which will make multimedia teaching. This will make multimedia teaching too dull so that the courseware programming board or the electronic version of the lecture notes will not be able to reflect the advantages of multimedia.

\section{Analysis of Results}

4.1. Multimedia Art Design Large-Database Design. The total gaze time for the different modules of the multimedia screen represents the sum of the duration of all gaze points within the subject's area of interest, and these data can reflect the extent to which the learner is processing the knowledge. It has become an important issue that must be properly resolved in teaching. In the research of this subject, the author tries to integrate the design concept of unit teaching "disintegrating into a whole" through the modern media art curriculum and strive to focus on teaching goals, optimize teaching content, and make students more comprehensive. A longer gaze time means that the area is more likely to attract the learner's attention or to be more difficult to process. Information that receives more 


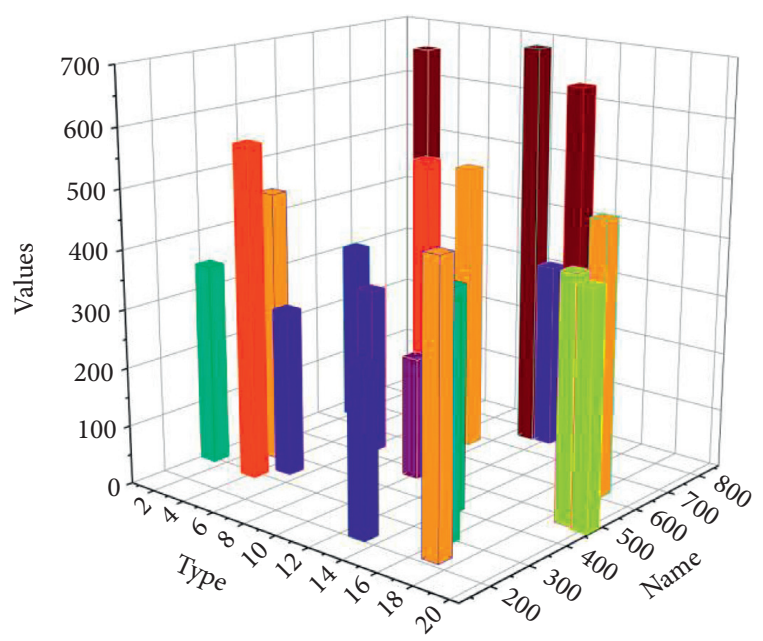

FIGURE 4: Clarity of instructional information presentation.

attention is more likely to be transmitted for further processing, as shown in Figure 5 below for the total gaze time data for the graphic area. It can be found in the statistics that the average total gaze time in the text area of the whole multimedia screen is significantly longer than the total gaze time of the diagram, which shows that the learners take significantly longer to process the knowledge information described in the text, but it does not mean that the diagram information is not important because the two kinds of information have different characteristics in themselves. Linear, abstract, and comprehensive are the distinctive features of textual language, which is a step-bystep complete expression of the complete content through an idea, requiring a strong logic, and therefore takes more time to read. Diagrams are three-dimensional and visual, and good diagrammatic information can be understood briefly, but it is condensed information and inevitably misses something about what is being expressed. The integrative learning style is more interested in diagrammatic information, and it can combine diagrammatic information more closely and take less time to read when studying.

Subjects with a more sequential style read the text following its logic as well as in step, and the overall time spent is relatively long, as will be reflected in the statistics of the hotzone data later. As a result, when designing multimedia images, we should ensure that the images and texts are well combined, as this can produce better visual effects and aid readers' memory.

To further explore how students with different learning styles allocate their attention in multimedia screens, this eye-movement experiment was conducted to compare eye-movement hot zones based on four dimensions of learning styles. The digitalization, virtualization, high-speed, technology, specialization, and internationalization of film and television art have been realized. Information technology has also had a profound impact on film and television creation, dissemination and consumption, and film and television aesthetics. The hotspot diagram allows us to visualize the subjects' gaze, revealing differences based on the size, location, and some color areas displayed in the hotspot zones in the diagram.
Two hot-zone comparison images with integrated very strong and serial type very weak and integrated very weak and serial type very strong are used, after which they are referred to as integrated and serial type. Integrated students had a slightly smaller gaze range than sequential students and significantly fewer hot zones than sequential subjects. The eye-movement data for the two learning styles were extracted, the average dwell time and the proportion of the two areas in the graphic text, and the average number and proportion of gaze points, as shown in Figure 6.

From Figure 6, it can be found that the total gaze time differs between the two types of students on the same screen, but the ratio of the gaze time on the picture to the time on the text is similar, and a similar pattern can be found by counting the other three dimensions of learning styles. When the narration, background music, and other information are presented to the learner's ears, the acquired information will be processed in the auditory-speech channel. Thus, different learning styles have a similar degree of relative processing of knowledge on the same picture, no picture would differentially affect different learning styles, and therefore multimedia pictures should have regular uniformity rules. Firstly, reference was made to existing questionnaires related to blended learning and multimedia screens, and Professor You Sewing's literature related to the language of multimedia screens and blended learning was also consulted and studied and then analyzed with due characteristics, and 13 variables were established from three aspects. After that, it was sent to the tutor and the students of educational technology to collect feedback for modification.

4.2. Results of the Optimization of the Hybrid Multimedia Art and Design Teaching Model. The distribution of positive emotions in both the university and social education levels showed a significant downward trend compared to the other segments, while emotions such as dull and dreary showed a significant increase, and the level of the distribution of negative emotions in these two segments was already higher than the level of positive emotions. There is a certain degree of art such as aesthetics, but more importantly, the effectiveness of its expression and its logical laws can be explored from the content level, the cognitive level, and the picturestructure level. Among the other emotion types, the distribution of emotions such as tedious, thrilling, and scary does not differ significantly from the distribution of the above three academic periods, except for unreal, which has increased. From the distribution of emotions, the design of teaching resources at the university and social education levels focuses more on the transmission of knowledge content, and the images are boring and have a single layout, which makes it difficult to convey positive emotions to learners in terms of visual characteristics. Figure 7 provides a visual representation of the distribution of emotions in each digital learning screen by school level.

The warm emotion type of digital learning screen is mainly present in teaching methods such as playing sound, animation, multimedia PPT, image, drawing, classroom, etc. 


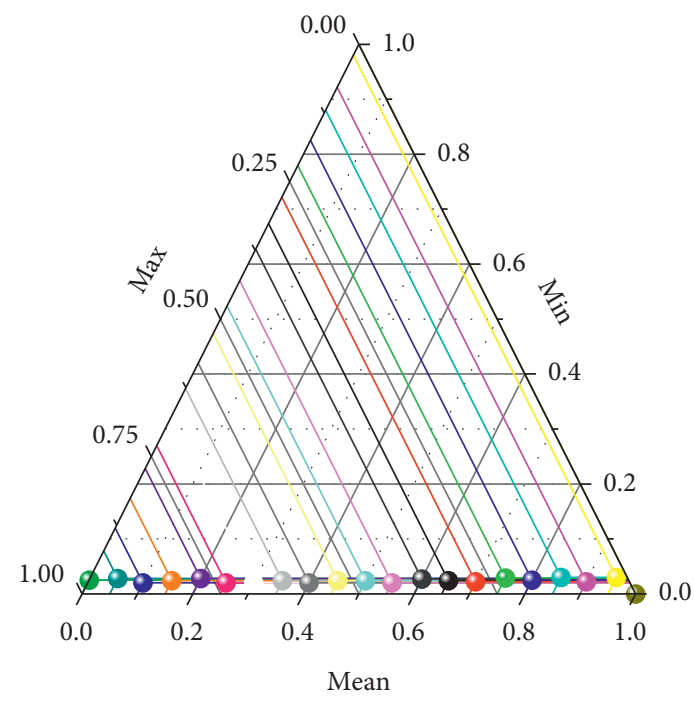

FIGURE 5: Description statistics of attention time.

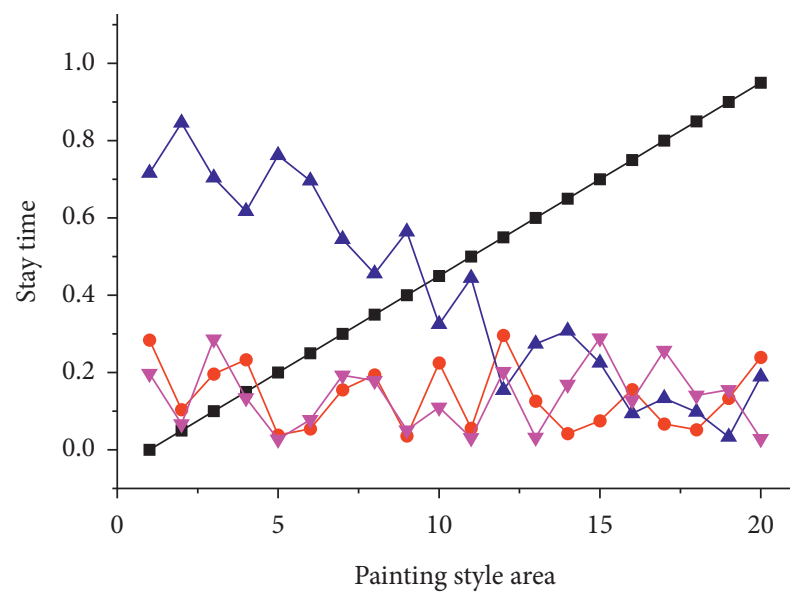

$$
\begin{aligned}
& \rightarrow \text { Stay time } \\
& \rightarrow \text { Fixation point ratio } \\
& \leftarrow \text { Percentage of residence time } \\
& \rightarrow-\text { Number of fixation points }
\end{aligned}
$$

FIgURE 6: Description of learning style interest area differences.

The level of warm emotion distribution of other types of resources is below $20 \%$. The distribution of warm emotions in videos and web pages is $6.25 \%$ and $1.68 \%$, and the lowest distribution of warm emotions in human-computer interaction is only $0.15 \%$. The cheerful emotion type is mainly found in the two teaching methods of animation and playing sound, and the distribution of other types of resources is also lower than $20 \%$, especially in the teacher and teaching content, video, web page, human-computer interaction, and classroom, the distribution level of cheerful emotion is less than $5 \%$. The lively emotion type is mainly found in the textbased teaching approach, probably because text-based resources enhance the visual characteristics of the resource due to a well-designed typographic layout and color scheme, which enhances the level of positive, lively emotion on the screen. The distribution of lively emotion in resources such as teacher's single lecture, teacher's teaching content, human-computer interaction, experimental training, and web page is low, all below $5 \%$. The funny emotion type is mainly distributed in the animation teaching method, and the animation-type resources are usually well designed with color and layout, which can convey good visual emotion characteristics. In contrast, the level of distribution of funny emotions is below $10 \%$ in all other types of images. The three emotions of exaggerated, humorous, and funny are not distributed high in all types of images, with the distribution of both humorous and funny emotions below $1 \%$, and most of the images have difficulty in reflecting the metaphorical characteristics of these two emotions.

Multimedia-assisted teaching in colleges and universities should start by changing the traditional teaching concept, establishing the teaching concept of students as the main body of the classroom, and carrying out classroom teaching from the perspective of facilitating students' learning and teachers' teaching while suggesting that teachers can apply a variety of teaching aids for diversified knowledge exchange. At the same time in the actual multimedia-assisted teaching teachers in addition to updating the concept of education and teaching, it should be clear that multimedia technology is only an auxiliary technical tool for teaching, rather than teaching. Multimedia-assisted teaching in colleges and universities should start by changing the traditional teaching concept, establishing the teaching concept of students as the main body of the classroom, and carrying out classroom teaching from the perspective of facilitating students' learning and teachers' teaching while suggesting that teachers can apply a variety of teaching aids for diversified knowledge exchange. Meanwhile, in the actual multimediaassisted teaching, teachers should make it clear that multimedia technology is only an auxiliary technical tool for teaching, not teaching, in addition to updating the concept of education and teaching, as shown in Figure 8.

Modern information technology is an important manifestation of science and technology, but today's society is a society in which economic capital occupies a major position. The main reason modern information technology has been promoted to such a high position and why it has been continuously improved and developed also lies in its value and the needs of the market, as well as the pursuit of profit, or the value orientation of contemporary society and film and art, which puts forward the information technology to a higher level of pursuit. When the value of a thing changes, the value of the thing or phenomenon related to it will also change accordingly. There is also a correlation, which refers to the fact that there is a certain relationship between things, which cannot be described by a fixed causal relationship. At the same time, although the correlation is not certain, it is regular. Thus, the face of technology or the manifestation of modern information technology is essentially a technology of the economy, even profit-oriented technology. The technology of economy or capital with science and technology, as the main means of benefiting the most, pushes technology to the center of the art of film and television. The 


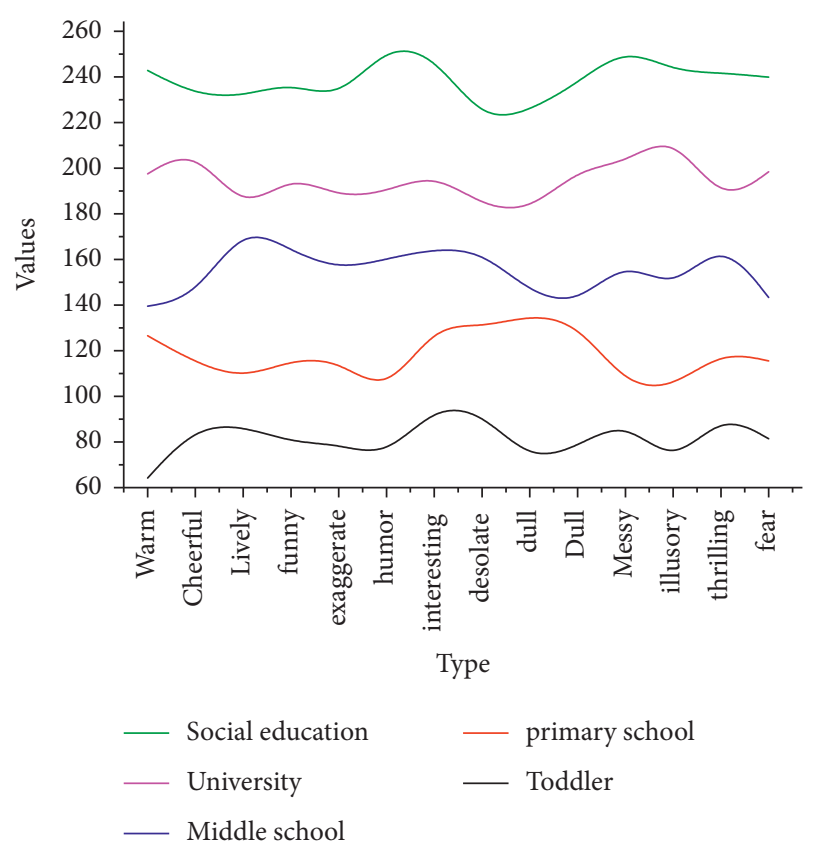

FIgURE 7: Distribution among school segments.

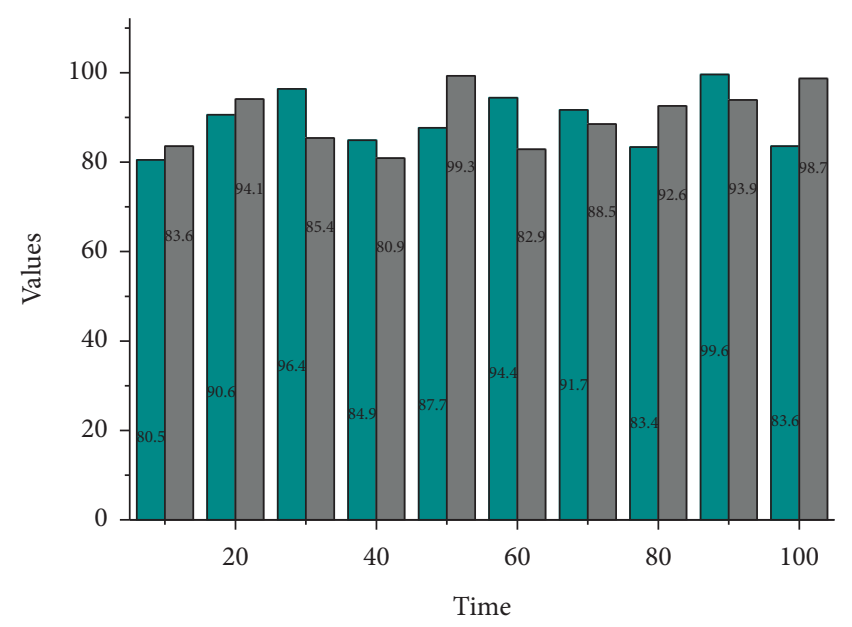

Teachers

Students

Figure 8: Evaluation results.

character of modern information technology, then, depends not only on the profitability of film and television art but also on the rational needs of modern film and television art that permeate the ultimate modern information technology, especially the scientific rationality of the needs of film and television art under mass aesthetics.

\section{Conclusion}

Art museum education in the context of the big data era faces more challenges and opportunities and opens a new chapter of development for art museum education. The booming development of big data has changed people's way of accessing information from active to passive, and in this transformation process, recommendation system has a crucial role. Recommendation algorithm is the key technology of recommendation system; the traditional recommendation algorithm still has the problems of cold start, high data sparsity, and so on. The prediction accuracy of the recommendation algorithm is an important criterion to measure the goodness of a recommendation system. Modern information technology has brought a great impact on the development of modern society because of its wide, fast, and technological communication, and for the development of film and television art, information technology not only makes its creative field and subject matter selection more extensive, but also it does limit the content of creation to reality. Modern information technology has broken the traditional time and space pattern, expanding the space and time of film and television production, making the impossible possible, and creating more infinite space and time possibilities through computerized postproduction of special effects. Modern information technology will also make the dissemination of film and television art more diversified and multichannel and obtain more infinite possibilities of film and television art, and the influence of film and television art will also be accompanied by the development of modern information technology and break through the traditional means of creation and expression so that the impossible becomes possible and dreams become reality.

\section{Data Availability}

The data used to support the findings of this study are included within the article.

\section{Conflicts of Interest}

The author does not have any possible conflicts of interest regarding the publication of this work.

\section{Acknowledgments}

This study was supported by Shanxi Province Education on Science Planning Project "Design and Application of Personalized Network Learning Space under the Concept of Maker Education" (No. GH-19165).

\section{References}

[1] G. Wang, X. Zheng, and Y. Ding, "Exploration on the talent cultivation path of tourism new media in the digital economy era," Converter, vol. 2021, no. 6, 319 pages, 2021.

[2] Y. Gong, "Application of virtual reality teaching method and artificial intelligence technology in digital media art creation," Ecological Informatics, vol. 63, Article ID 101304, 2021.

[3] N. Wiltz and G. P. Wilson, "An inquiry into children's reading in one urban school using SRA reading mastery (direct instruction)," Journal of Literacy Research, vol. 37, no. 4, pp. 493-528, 2005.

[4] G. J. Hwang and Q. K. Fu, "Advancement and research trends of smart learning environments in the mobile era," International Journal of Mobile Learning and Organisation, vol. 14, no. 1, pp. 114-129, 2020. 
[5] C. Meng-yue, L. Dan, and W. Jun, "A study of college English culture intelligence-aided teaching system and teaching pattern," English Language Teaching, vol. 13, no. 3, pp. 77-83, 2020.

[6] S.-T. Xie, Z.-B. He, Q. Chen, R.-X. Chen, Q.-Z. Kong, and C.-Y. Song, "Predicting learning behavior using log data in blended teaching," Scientific Programming, vol. 2021, Article ID 4327896, 14 pages, 2021.

[7] Y. Jiang, K. Zhao, K. Xia et al., "A novel distributed multitask fuzzy clustering algorithm for automatic MR brain image segmentation," Journal of Medical Systems, vol. 43, no. 5, pp. 118-118:9, 2019.

[8] X. Gao and Y. Wang, "Optimized integration of traditional folk culture based on DSOM-FCM," Personal and Ubiquitous Computing, vol. 24, no. 2, pp. 273-286, 2020.

[9] D. Lande, I. Subach, and A. Puchkov, "A system for analysis of big data from social media," Information and Security: An International Journal, vol. 47, no. 1, pp. 44-61, 2020.

[10] N. Li, "Construction of landscape architecture art design based on streaming media data processing," International Journal of Arts and Technology, vol. 12, no. 4, pp. 335-351, 2020.

[11] J. A. Patel and P. Sharma, "Online analytical processing for business intelligence in big data," Big Data, vol. 8, no. 6, pp. 501-518, 2020.

[12] C. Østerlund, M. H. Jarrahi, M. Willis, and K. Boyd, "Artificial intelligence and the world of work, a co-constitutive relationship," Journal of the Association for Information Science and Technology, vol. 72, no. 1, pp. 128-135, 2021.

[13] M. Ramsgaard Thomsen, P. Nicholas, M. Tamke, S. Gatz, Y. Sinke, and G. Rossi, "Towards machine learning for architectural fabrication in the age of industry 4.0," International Journal of Architectural Computing, vol. 18, no. 4, pp. 335-352, 2020.

[14] S. Kocdar, A. Bozkurt, and T. Goru Dogan, "Engineering through distance education in the time of the fourth industrial revolution: reflections from three decades of peer reviewed studies," Computer Applications in Engineering Education, vol. 29, no. 4, pp. 931-949, 2021.

[15] S. Sariani and M. E. Khairat, "An optimization of language learning in writing through E-learning: encountering covid19 pandemic," International Journal of Language Education, vol. 5, no. 1, pp. 528-541, 2021.

[16] Z. Dafir, Y. Lamari, and S. C. Slaoui, "A survey on parallel clustering algorithms for big data," Artificial Intelligence Review, vol. 54, no. 4, pp. 2411-2443, 2021.

[17] S. D. Arul and M. Iyapparaja, "Social internet of things using big data analytics and security aspects-a review," Electronic Government, An International Journal, vol. 16, no. 1-2, pp. 137-154, 2020.

[18] D. Xiaofeng, "Application of deep learning and artificial intelligence algorithm in multimedia music teaching," Journal of Intelligent and Fuzzy Systems, vol. 38, no. 6, pp. 7241-7251, 2020.

[19] M. Li, "A study on the influence of non-intelligence factors on college students' English learning achievement based on C4.5 algorithm of decision tree," Wireless Personal Communications, vol. 102, no. 2, pp. 1213-1222, 2018.

[20] J. Tian and G. He, "The five-in-one teaching mode in the teaching of engineering courses," Computer Applications in Engineering Education, vol. 28, no. 6, pp. 1683-1695, 2020.

[21] Y. Chen, "A survey on industrial information integration 2016-2019," Journal of Industrial Integration and Management, vol. 5, no. 1, pp. 33-163, 2020. 